\title{
Forbidden Vibrational Transitions in Cold Molecular Ions: Experimental Observation and Potential Applications
}

\author{
Matthias Germann $\S^{\star a}$, Xin Tong ${ }^{\text {ab }}$, and Stefan Willitsch ${ }^{\star a}$
}

§SCS-DSM Award for best poster presentation

\begin{abstract}
A range of interesting fundamental scientific questions can be addressed by high-precision molecular spectroscopy. A promising way towards this goal is the measurement of dipole-forbidden vibrational transitions in molecular ions. We have recently reported the first such observation in a molecular ion. ${ }^{[1]}$ Here, we give an overview of our method and our results as well as an outlook on potential future applications.
\end{abstract}

Keywords: Cold molecular ions · Forbidden transitions · Precision spectroscopy · Vibrational spectroscopy

\section{Introduction}

It is a well-known tenet in the field of molecular spectroscopy that homonuclear diatomic molecules, such as $\mathrm{H}_{2}$ or $\mathrm{N}_{2}$, do not show a vibrational spectrum because their electric-dipole moment vanishes for all internuclear separations and thus does not change upon vibration. Though this statement is true within the so-called 'electric-dipole approximation', higher order terms do exist in the multipole expansion of the interaction of molecules with radiation, e.g. the electric-quadrupole term, that give rise to very weak 'forbidden' transitions in their rovibrational spectrum. ${ }^{[2,3]}$ Indeed, such forbidden lines have been observed for a couple of diatomic molecules. The first observation dates back over half a century and was made by Herzberg in an absorption spectrum of $\mathrm{H}_{2} \cdot{ }^{[4]}$ Because of the extreme weakness of these transitions, an equivalent absorption path length of several kilometers was needed for this experiment. Later, similar measurements on electric-quadrupole rotation-vibration spectra of other molecules such as $\mathrm{N}_{2}$ and $\mathrm{O}_{2}{ }^{[5,6]}$ have been performed. In more recent times, the technique of cavity ring-down spectroscopy which, too, is based on very long absorption path lengths, was employed for such measurements - some of

\footnotetext{
$\overline{{ }^{*} \text { Correspondence: }}$ M. Germann, Prof. Dr. S. Willitsch ${ }^{a}$ E-Mail: matthias.germann@unibas.ch, stefan.willitsch@unibas.ch

aDepartement Chemie, Universität Basel Klingelbergstrasse 80

$\mathrm{CH}-4056$ Basel

bState Key Laboratory of Magnetic Resonance and Atomic and Molecular Physics

Wuhan Institute of Physics and Mathematics

Chinese Academy of Sciences,

Wuhan 430071, China
}

them also exhibiting a remarkable precision (see for example ref. [7]).

The weakness of such 'forbidden' lines does not just represent an experimental obstacle but rather provides a remarkable potential, as it is associated with an extremely narrow natural linewidth and - correspondingly - a very long lifetime of the excited state of such a transition. Estimates show lifetimes of several months and linewidths reaching down to the nanohertz range.[1] Indeed, experiments on forbidden electronic transitions in atomic ions allowed some of the most precise measurements in science in general. These include experiments such as the comparison of two transition frequencies between a single $\mathrm{Al}^{+}$ and a single $\mathrm{Hg}^{+}$ion ${ }^{[8]}$ with a precision of $10^{-17}$ or the precise comparison of the transition frequencies of two different forbidden transitions in a single $\mathrm{Yb}^{+}$ion. ${ }^{[9]}$ Such high-precision measurements allow the examination of subtle fundamental effects such as the relativistic time dilation of an atomic clock within the earth's gravitational field ${ }^{[10]}$ or a possible time variation of the fine-structure constant. ${ }^{[8]}$ Besides the narrow natural linewidth of forbidden transitions, these experiments also exploited several advantages of using ions for such studies: Because of their charge, ions may be electrically trapped and thereby isolated from the environment as well as 'sympathetically' cooled. Sympathetic cooling means that a metrologically favorable ion (such as $\mathrm{Al}^{+}$), which can hardly or not at all be laser cooled, is cooled via the exchange of kinetic energy with a cotrapped, easily laser-coolable ion of another species (e.g. $\left.\mathrm{Be}^{+[8]}\right)$. Cooling is essential to reduce Doppler shifts and to allow long interrogation times.
However, unlike the case for atomic ions or for neutral molecules, until recently there have been no reports of measurements of forbidden transitions in molecular ions to the best of our knowledge. Therefore, the above-mentioned advantages of ions have thus far not been exploited in the field of molecular spectroscopy. Because of their charge and their high reactivity, high number densities or long absorption path lengths cannot be achieved with molecular ions and thus the techniques used this far for the observation of forbidden transitions in neutral molecules are not applicable. Furthermore, the schemes devised to study transitions in single atomic ions are not directly adaptable to molecular ions due to their rich structure of quantum states (electronic, vibrational, rotational), which prohibits schemes based on closed optical cycling transitions for cooling, state-preparation and detection.

\section{Electric-quadrupole Rotational- vibrational Transitions in $\mathrm{N}_{2}^{+}$}

We have recently reported the first observation of a forbidden rotationalvibrational transition in a molecular ion. ${ }^{[1]}$ Instead of a long absorption path, our approach is based on a long interrogation time in combination with a highly sensitive detection technique to spot even single absorption events. Specifically, we have observed the fundamental infrared (IR) S(0) transition in the prototypical homonuclear diatomic ion $\mathrm{N}_{2}^{+}$, i.e. the transition from the vibrational and rotational ground state $\left(V^{+\prime \prime}\right.$ $\left.=0, N^{+\prime}=0\right)$ to the second rotationally excited level $\left(N^{+\prime}=2\right)$ of the first vibrationally excited state $\left(v^{+\prime}=1\right)$ within the electronic 
ground state (see Fig. 1 for a level scheme of $\mathrm{N}_{2}^{+}$). Here, $v^{+}$and $N^{+}$denote the vibrational and rotational quantum numbers of the ion, and " and 'refer to the lower and upper level of the transition, respectively. Additional to the vibrational and rotational structure, $\mathrm{N}_{2}^{+}$also shows fine and hyperfine structure. The electronic ground state $\mathrm{X}^{2} \Sigma_{\mathrm{g}}{ }^{+}$of $\mathrm{N}_{2}{ }^{+}$may be well described by the Hund's case $b_{\beta J}$ coupling scheme, ${ }^{[11]}$ meaning that the rotational angular momentum of the molecule $\mathbf{N}$ is first coupled with its $\operatorname{spin} \mathbf{S}$ to form $\mathbf{J}=\mathbf{N}+\mathbf{S}$. As we are considering a doublet state, we have $S=1 / 2$ and rotational excited states are split into two spin-rotation components. Thereafter, $\mathbf{J}$ is coupled to the nuclear spin I to form the total angular momentum $\mathbf{F}=\mathbf{J}+\mathbf{I}$. For ${ }^{14} \mathrm{~N}_{2}{ }^{+}$ and the states considered here, we have $I=0$ or $I=2$. In the latter case, the energy levels are split further into a number of hyperfine levels. Electric-quadrupole transitions are governed by the selection rule $\Delta F=0, \pm 1, \pm 2$ which is accompanied by the propensity rule $\Delta F=\Delta J=\Delta N$ (similar as in the case of dipole-allowed transitions $\left.^{[11]}\right)$. As indicated in Fig. 1, there are three transitions obeying this rule.

\section{Experiment}

As mentioned above, our experiment is based on a long interrogation time of the forbidden transition. For this reason, the $\mathrm{N}_{2}{ }^{+}$ions were stored in an ion trap that forms the heart of our experimental setup (see Fig. 2). The trap consists of four cylindrical electrodes arranged as the four long edges of an elongated cuboid. Sinusoidally oscillating voltages with an amplitude of $120 \mathrm{~V}$ and a frequency of $3.2 \mathrm{MHz}$ applied to the rods exert, on time average, a confining force on the ions and prevent their escape from the trap in a radial direction. Additional dc voltages of $1.5 \mathrm{~V}$ applied to isolated end sections of the rods provide an axial trapping force. The trap was loaded with typically $20-25 \mathrm{~N}_{2}{ }^{+}$ions by photoionization of neutral $\mathrm{N}_{2}$ molecules from a pulsed, doubly-skimmed molecular beam. For photoionization, a two-color [2+1'] resonance-enhanced multiphoton ionization (REMPI) scheme was employed. ${ }^{[12,13]}$ By setting the energy of the third photon just above the lowest ionization threshold of $\mathrm{N}_{2}$, the $\mathrm{N}_{2}^{+}$ions were only produced in the $\mathrm{X}^{2} \Sigma_{\mathrm{g}}^{+}, v^{+\prime \prime}=0, N^{+\prime \prime}=0$ rovibronic ground state and were thus initialized for the subsequent measurement of the $S(0)$ rotational-vibrational transition. The $\mathrm{N}_{2}{ }^{+}$ ions were sympathetically cooled with cotrapped atomic $\mathrm{Ca}^{+}$ions to temperatures in the millikelvin range. Unlike $\mathrm{N}_{2}^{+}, \mathrm{Ca}^{+}$ exhibits an almost closed cycling transition at $397 \mathrm{~nm}\left(4^{2} \mathrm{~S}_{1 / 2} \rightarrow 4^{2} \mathrm{P}_{3 / 2}\right)$, which was used for laser cooling. A small fraction of

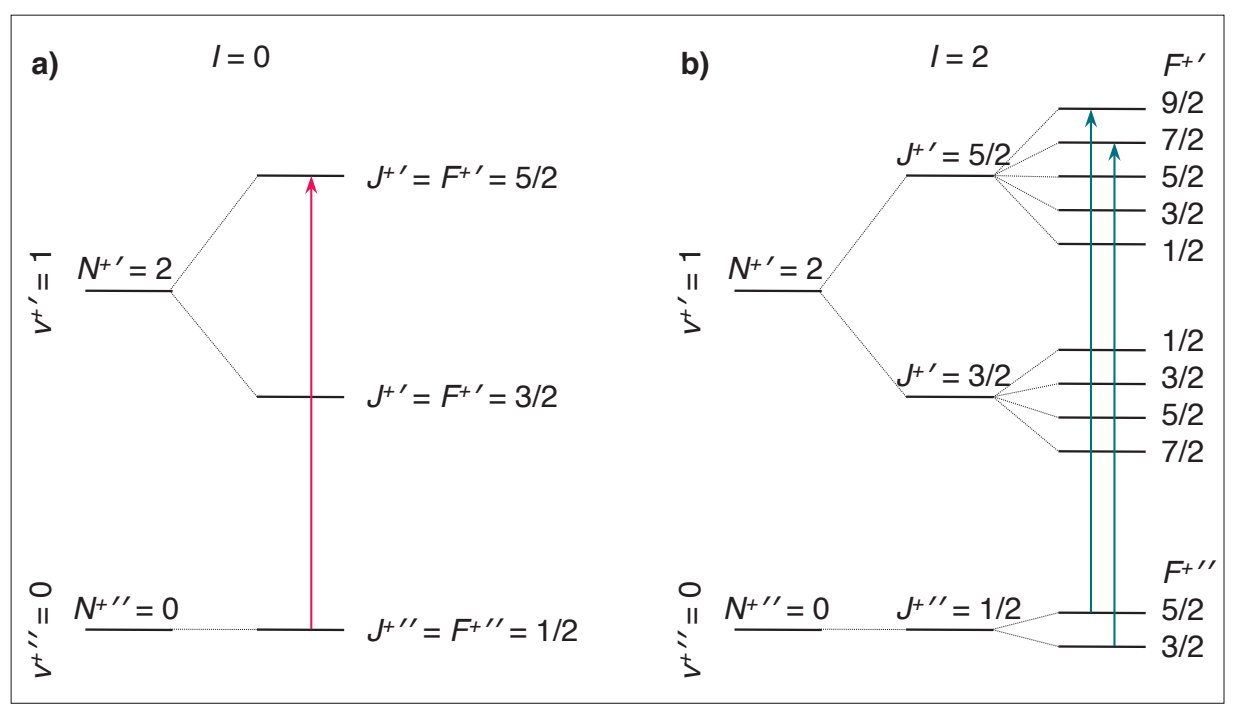

Fig. 1. Scheme of the energy levels of the $S(0)$ electric-quadrupole rotational-vibrational transition in $\mathrm{N}_{2}{ }^{+}$. a) Vanishing total nuclear spin ( $=0$ nuclear spin manifold): The rotational excited level is split into two spin-rotation components (fine structure). b) For the $I=2$ nuclear spin manifold those are further split, resulting in the hyper-fine structure. The transitions obeying the propensity rule $\Delta F=\Delta J=\Delta N=2$ are indicated by vertical arrows (reproduced from ref. [1]). the population in the $4^{2} \mathrm{~S}_{1 / 2}$ state decays to the metastable $3^{2} \mathrm{D}_{3 / 2}$ state. From there, it was repumped with an additional laser at $866 \mathrm{~nm}$. Following cooling, the ions form an ordered structure in the trap known as a Coulomb crystal. The fluorescence light of the $\mathrm{Ca}^{+}$ions was collected with a microscope and detected by a CCD camera to image the crystal. The $\mathrm{N}_{2}{ }^{+}$ions do not fluoresce and are not directly visible. Because of their lighter mass, they form a string along the trap axis where they appeared as a dark core (see Fig. 3 ). The $\mathrm{S}(0)$ electricquadrupole transition in $\mathrm{N}_{2}^{+}$was excited with mid-infrared radiation produced by a quantum cascade laser (QCL). The QCL was operated at a wavelength around $4.6 \mu \mathrm{m}$ and delivered an effective power of about $170 \mathrm{~mW}$, which was irradiated on the Coulomb crystal for about $2 \mathrm{~min}$ in a usual experimental cycle.

For the detection of vibrationally excited $\mathrm{N}_{2}^{+}$ions, a charge transfer (CT) reaction with Ar atoms was used. Vibrationally excited $\mathrm{N}_{2}{ }^{+}$react with $\mathrm{Ar}$ according to

\section{$\mathrm{N}_{2}^{+}\left(v^{+} \geq 1\right)+\mathrm{Ar} \rightarrow \mathrm{N}_{2}+\mathrm{Ar}^{+}$}

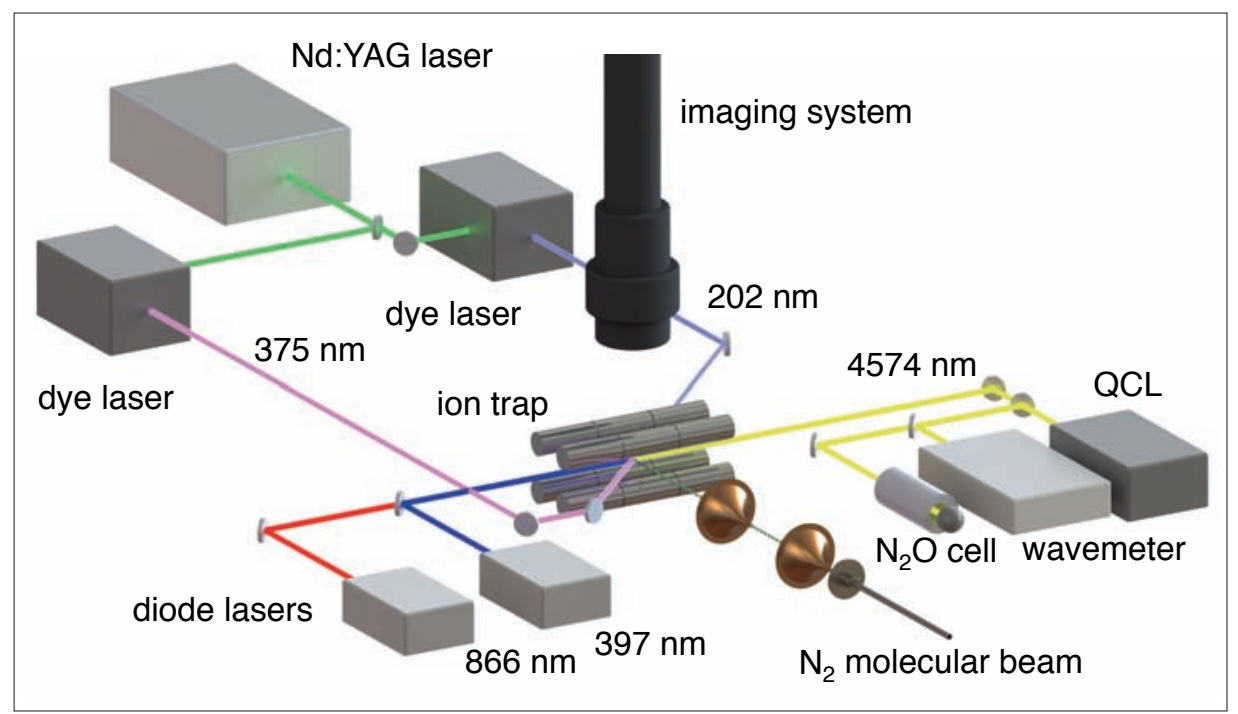

Fig. 2. Experimental setup for the observation of dipole-forbidden vibrational transitions in $\mathrm{N}_{2}{ }^{+}$. The heart of the experiment is formed by a linear radiofrequency ion trap. The trap is loaded by state-selective ionization of neutral $\mathrm{N}_{2}$ from a molecular beam. $\mathrm{N}_{2}+$ ions are sympathetically cooled by simultaneously trapped $\mathrm{Ca}^{+}$ions that are laser cooled by two diode lasers $(397 \mathrm{~nm}$ and $866 \mathrm{~nm}$ ). The $\mathrm{N}_{2}{ }^{+}$and $\mathrm{Ca}^{+}$ions form a bicomponent Coulomb crystal that is imaged with a CCD camera attached to a microscope. Dipole-forbidden vibrational transitions are driven by IR radiation from a quantum cascade laser (QCL) (adapted from ref. [1]). 


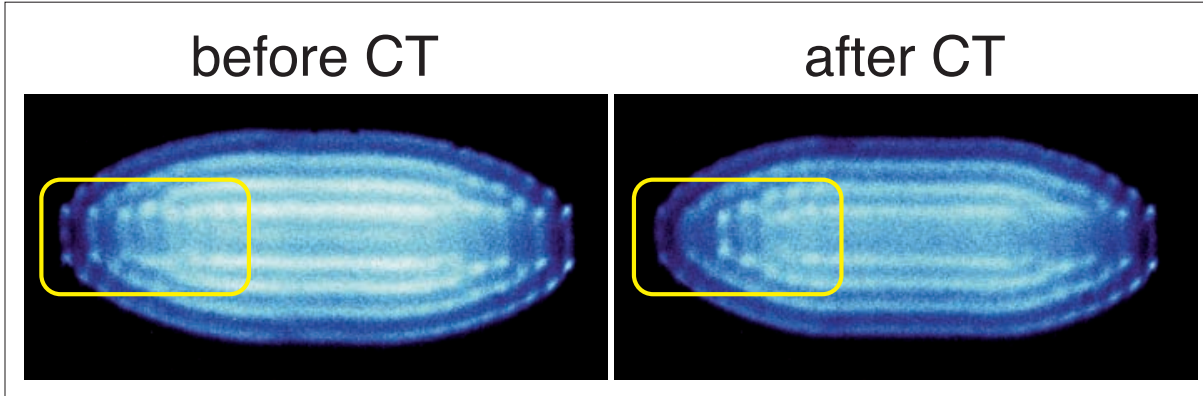

Fig. 3. Fluorescence images of bicomponent $\mathrm{Ca}^{+} / \mathrm{N}_{2}{ }^{+}$Coulomb crystals taken during one experimental cycle before and after IR irradiation and CT detection of $\mathrm{N}_{2}{ }^{+}$ions. The $\mathrm{N}_{2}{ }^{+}$ions appear as a non-fluorescing core in the centre of the crystals. Slight changes in the structure of the core indicate the removal of $\mathrm{N}_{2}{ }^{+}$ions by CT reactions following their excitation (see highlighted regions) (adapted from ref. [1]).

The neutral $\mathrm{N}_{2}$ molecules formed in this reaction were lost from the trap, whereas the newly formed $\mathrm{Ar}^{+}$ions diffused into the $\mathrm{Ca}^{+}$Coulomb crystal because of the similar masses of these two species. The reaction was thus observable as a decrease of the dark core in the Coulomb crystal (see Fig. 3 ). For ions in the vibrational ground state, the above reaction is endothermic and thus suppressed. [14] The fraction of lost $\mathrm{N}_{2}{ }^{+}$ions therefore indicates the amount of vibrationally excited $\mathrm{N}_{2}^{+}$ions after IR irradiation at a certain wavelength. To determine the number of $\mathrm{N}_{2}^{+}$ions in a Coulomb crystal, the experimental images were compared to synthetic ones obtained from numerical simulations of the ion dynamics. ${ }^{[1]}$ With this method, a determination of the number of vibrationally excited $\mathrm{N}_{2}^{+}$ions with an accuracy of \pm 1 was achieved.

\section{Results and Discussion}

By repeating the experimental procedure described above for different IR excitation frequencies and plotting the number of CT events as a function of the excitation frequency, we obtained a spectrum of hyperfine components of the $S(0)$ line shown in Fig. 4. The spectrum shows two well separated peaks: A broader one at a lower frequency and a narrower one at a roughly $200 \mathrm{MHz}$ higher frequency. The theoretically predicted line positions of the three lines obeying the aforementioned propensity rule are indicated by vertical lines in Fig. 4. These predictions are based on known molecular constants for $\mathrm{N}_{2}^{+} \mathrm{ob}-$ tained from spectra of dipole-allowed $\mathrm{rf}$ and electronic transitions ${ }^{[11,15]}$ and were calculated by diagonalizing the fine and hyperfine structure Hamiltonian from ref. [11] as well as adding the corresponding rotational and vibrational term energies. ${ }^{[15]}$ Comparison with these predictions allows us to assign the narrower and higher-frequency peak to the $F^{+\prime \prime}=3 / 2 \rightarrow F^{+\prime}=7 / 2$ transition of the $I=2$ nuclear spin manifold.
The observed width of this peak (about 19 $\mathrm{MHz}$ FWHM) is attributed to the bandwidth of the QCL. The natural linewidth is estimated to be several orders of magnitude smaller (approximately nanohertz ${ }^{[1]}$ ) and the Doppler width at the temperature of the ions amounts to approximately 1.3 MHz. The lower-frequency peak is assigned to the overlap of the $F^{+\prime \prime}=5 / 2 \rightarrow$ $F^{+\prime}=9 / 2$ transition of the $I=2$ and the $J^{+\prime \prime}=1 / 2 \rightarrow J^{+\prime}=5 / 2$ transition of the $I=0$ ref. [1]). nuclear spin isomers. As seen in Fig. 4, the measured line positions all appear at slightly lower frequencies than predicted. To put this discrepancy into perspective, we note that our predictions are based on vibrational and rotational constants previously determined from electronic spectra. Considering the spread of their values among different studies in the literature on the level of accuracy achieved in our experiment, this deviation seems acceptable. However, the relative positions of the three lines - given by the hyperfine splittings of the respective levels - are satisfactorily reproduced in our spectrum. Thus the observed agreement between predictions and measurements seems satisfactory.

\section{Conclusion and Outlook}

We have for the first time observed electric-dipole-forbidden rotation-vibration transitions in a molecular ion. Such observations open up new applications in the field of high-precision spectroscopy and frequency metrology. High-precision measurement on molecules have, for example, been used to study questions such

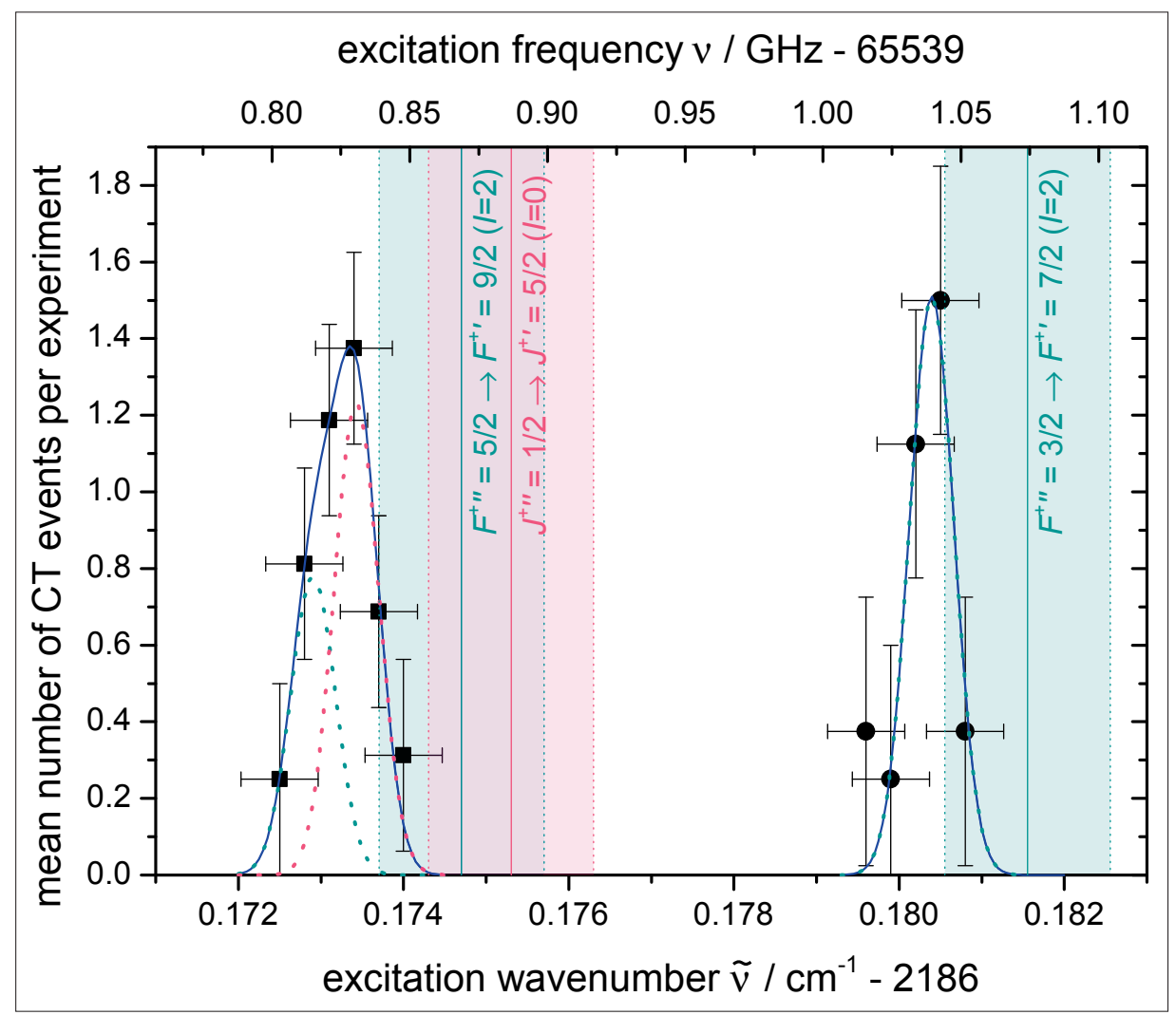

Fig. 4. Spectrum of hyperfine components of the $\mathrm{S}(0)$ electric-quadrupole transition in ortho- $\mathrm{N}_{2}{ }^{+}$ represented as the mean number of CT events per experimental cycle as a function of the excitation frequency. Data points marked with squares (circles) were averaged over 16 (8) experiments. The horizontal and vertical error bars correspond to the relevant $1 \sigma$ statistical uncertainties. The dotted lines represent Gaussian fits of the three spectral transitions to the data points and the solid blue line their sum. The solid green (red) vertical lines show predicted positions of hyperfine transitions of the $I=2(I=0)$ nuclear spin isomer indicated in Fig. 1, based on spectroscopic constants from the literature. The shaded areas represent their $1 \sigma$ uncertainties (reproduced from 
as a possible variation of fundamental physical constants, ${ }^{[16]}$ the existence of yet unknown fundamental interactions ${ }^{[17]}$ or to constrain the magnitude of the electron's electric dipole moment.[18] Many of these questions can suitably be studied with the rotation-vibration spectra of molecules. Molecular rotation-vibration transitions show, e.g., a more prominent dependence on a possible variation of the proton to electron mass ratio $m_{\mathrm{p}} / m_{\mathrm{e}}$ than atomic term energies ${ }^{[19]}$ and they also provide a way to search for unknown long-range hadron-hadron interactions. [17] Indeed, rotation-vibration transitions in $\mathrm{N}_{2}^{+}$have been proposed to search for $m_{\mathrm{p}} / m_{\mathrm{e}}$ variations. ${ }^{[19]}$ Obviously, the accuracy and precision of the measurements needs to be improved for such applications. To that end, radiation sources with a smaller bandwidth as well as new non-destructive detection schemes based on quantum logic applicable to single-molecule experiments should be employed. ${ }^{[20]}$ Looking even further into the future, experiments with polyatomic molecular ions could be envisioned that would allow studying the influence of the parity-violating weak interaction on chiral molecules ${ }^{[21]}$ and its possible ramification on the homochirality found in biomolecules. ${ }^{[22]}$

\section{Acknowledgements}

We would like to thank D. Wild, M. Grischa, P. Knöpfel, G. Holderied and A. Johnson for technical support. This work was supported by the University of Basel, the Swiss National Science Foundation as part of the National Centre of Competence in Research, Quantum Science \& Technology (NCCRQSIT) and the European Commission under the Seventh Framework Programme FP7 GA 607491 COMIQ.

Received: January 23, 2015

[1] M. Germann, X. Tong, S. Willitsch, Nat. Phys. 2014, 10,820 .

[2] D. Papoušek, Collect. Czechoslov. Chem. Commun. 1989, 54, 2555.

[3] B. H. Bransden, C. J. Joachain, 'Physics of Atoms and Molecules', 2nd ed., Prentice Hall, Harlow, 2003

[4] G. Herzberg, Nature 1949, 163, 170.

[5] D. Reuter, D. E. Jennings, J. W. Brault, J. Mol. Spectrosc. 1986, 115, 294.

[6] J. Reid, R. L. Sinclair, A. M. Robinson, A. R. W. McKellar, Phys. Rev. A 1981, 24, 1944.

[7] C.-F. Cheng, Y. R. Sun, H. Pan, J. Wang, A.W. Liu, A. Campargue, S.-M. Hu, Phys. Rev. A 2012, 85, 024501.

[8] T. Rosenband, D. B. Hume, P. O. Schmidt, C. W. Chou, A. Brusch, L. Lorini, W. H. Oskay, R. E. Drullinger, T. M. Fortier, J. E. Stalnaker, S. A. Diddams, W. C. Swann, N. R. Newbury, W. M, Itano, D. J. Wineland, J. C. Bergquist, Science 2008, 319, 1808.
[9] R. M. Godun, P. B. R. Nisbet-Jones, J. M. Jones, S. A. King, L. A. M. Johnson, H. S. Margolis, K. Szymaniec, S. N. Lea, K. Bongs, P. Gill, Phys. Rev. Lett. 2014, 113, 210801.

[10] C. W. Chou, D. B. Hume, T. Rosenband, D. J. Wineland, Science 2010, 329, 1630.

[11] N. B. Mansour, C. Kurtz, T. C. Steimle, G. L. Goodman, L. Young, T. J. Scholl, S. D. Rosner, R. A. Holt, Phys. Rev. A 1991, 44, 4418.

[12] X. Tong, A. H. Winney, S. Willitsch, Phys. Rev. Lett. 2010, 105, 143001.

[13] X. Tong, D. Wild, S. Willitsch, Phys. Rev. A 2011, 83, 023415 .

[14] S. Schlemmer, T. Kuhn, E. Lescop, D. Gerlich, Int. J. Mass Spectrom. 1999, 185, 589.

[15] F. Michaud, F. Roux, S. P. Davis, A.-D. Nguyen, C. O. Laux, J. Mol. Spectrosc. 2000, 203, 1.

[16] P. Jansen, H. L. Bethlem, W. Ubachs, J. Chem. Phys. 2014, 140, 010901.

[17] E. J. Salumbides, J. C. J. Koelemeij, J. Komasa, K. Pachucki, K. S. E. Eikema, W. Ubachs, Phys. Rev. D 2013, 87, 112008.

[18] The ACME Collaboration, J. Baron, W. C. Campbell, D. DeMille, J. M. Doyle, G. Gabrielse, Y. V. Gurevich, P. W. Hess, N. R. Hutzler, E. Kirilov, I. Kozyryev, B. R. O'Leary, C. D. Panda, M. F. Parsons, E. S. Petrik, B. Spaun, A. C. Vutha, A. D. West, Science 2014, 343, 269.

[19] M. Kajita, G. Gopakumar, M. Abe, M. Hada, M. Keller, Phys. Rev. A 2014, 89, 032509.

[20] J. Mur-Petit, J. J. García-Ripoll, J. Pérez-Ríos, J. Campos-Martínez, M. I. Hernández, S. Willitsch, Phys. Rev. A 2012, 85, 022308.

[21] M. Quack, J. Stohner, M. Willeke, Annu. Rev. Phys. Chem. 2008, 59, 741.

[22] M. Quack, Angew. Chem. Int. Ed. 2002, 41, 4618. 